\title{
GEMS X-ray Polarimeter performance simulations
}

\author{
Wayne H. Baumgartner ${ }^{a, b}$, Tod Strohmayer ${ }^{b}$, Tim Kallman ${ }^{b}$, \\ J. Kevin Black ${ }^{c, b}$, Joanne Hill ${ }^{b}$, Jean Swank ${ }^{b}$ \\ ${ }^{a}$ University of Maryland Baltimore County, 1000 Hilltop Circle, Baltimore, MD, USA; \\ ${ }^{b}$ NASA/GSFC, Code 662, Greenbelt, MD, USA; \\ ${ }^{c}$ Rock Creek Scientific, Washington, DC, USA
}

\begin{abstract}
The Gravity and Extreme Magnetism Small explorer (GEMS) is an X-ray polarization telescope selected as a NASA small explorer satellite mission. The X-ray Polarimeter on GEMS uses a Time Projection Chamber gas proportional counter to measure the polarization of astrophysical X-rays in the $2-10 \mathrm{keV}$ band by sensing the direction of the track of the primary photoelectron excited by the incident X-ray.

We have simulated the expected sensitivity of the polarimeter to polarized X-rays. We use the simulation package Penelope to model the physics of the interaction of the initial photoelectron with the detector gas and to determine the distribution of charge deposited in the detector volume. We then model the charge diffusion in the detector, and produce simulated track images. Within the track reconstruction algorithm we apply cuts on the track shape and focus on the initial photoelectron direction in order to maximize the overall sensitivity of the instrument. Using this technique we have predicted instrument modulation factors $\mu_{100}$ for $100 \%$ polarized X-rays ranging from $10 \%$ to over $60 \%$ across the $2-10 \mathrm{keV}$ X-ray band.
\end{abstract}

We also discuss the simulation program used to develop and model some of the algorithms used for triggering, and energy measurement of events in the polarimeter.

Keywords: GEMS, X-ray, Polarimetry

\section{INTRODUCTION}

The Micropattern Time Projection Chamber ${ }^{1,2,3,4}$ is at the heart of the polarimeter used for the GEMS mission ${ }^{5}$. It operates in the 2-10 keV X-ray band, and consists of a gas proportional counter filled with 190 Torr DiMethyl Ether (DME). The drift direction in the detector is perpendicular to the incident direction of the photons focused by the X-ray mirror, which allows for very long photon path lengths in the detector and high quantum efficiency. Multiplication of the charge deposited by the initial photoelectron is provided by a Gas Electron Multiplier (GEM); and the signal is read out by a set of 128 one dimensional strips coupled to the GEM with their long directions parallel to the initial photon direction. When regular time samples of the signals present on the strips are taken, a two-dimensional image can be made of the charge track deposited by the initial photoelectron. The initial direction of the photoelectron is closely correlated with the polarization of the incident photon, and analysis of many events can yield information on the polarization of the X-ray source.

A cross section diagram of a GEMS polarimeter is shown in Figure 1. The photons arrive from the left, interact with the gas in the detector depositing charge, which then drifts down to the GEM where the charge is multiplied, collected by the strips, and read by the APV25 ASIC. Figure 2 further illustrates how the charge read out sequentially in this fashion forms a two dimensional image of the initial photoelectron track.

A typical observation will record many events and derived track directions, on the order of one million or more for an astrophysical observation. A histogram of the derived track directions for a single observation of

Author E-mail: whbaumga@gmail.com 


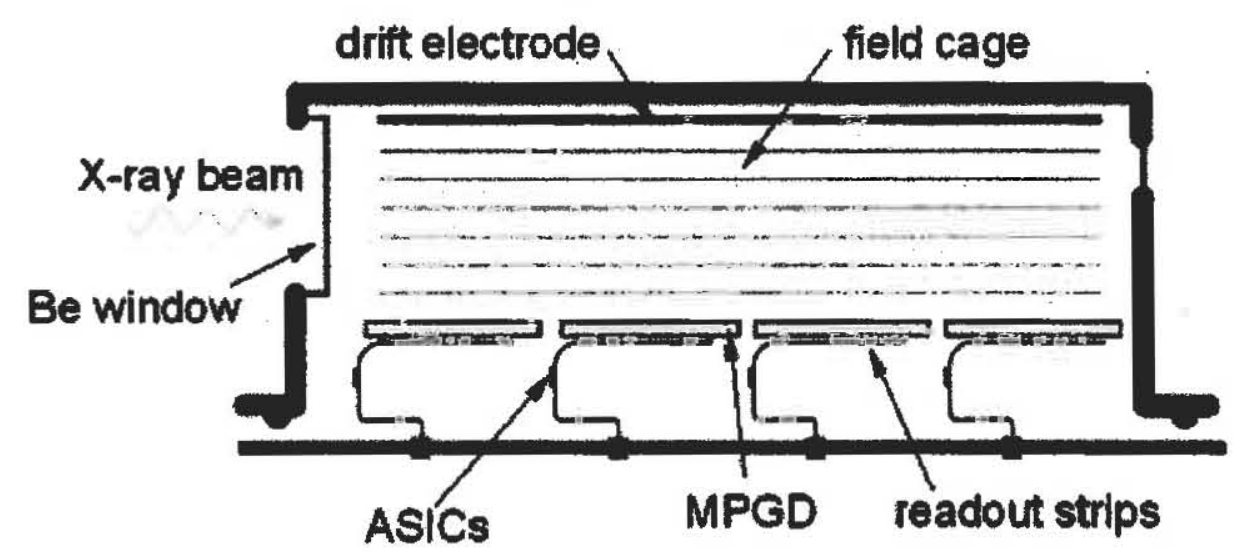

Figure 1. Diagram of GEMS Time Projection Chamber polarimeter.

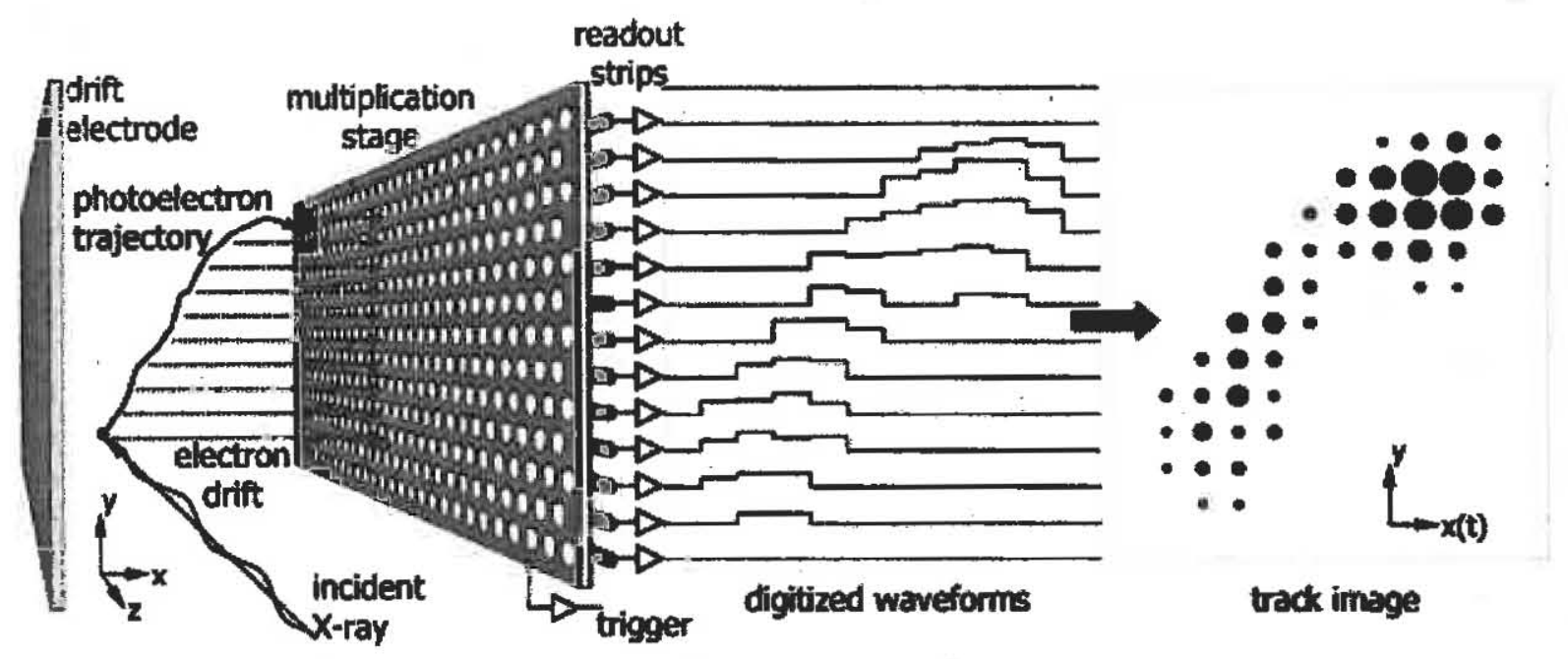

Figure 2. Diagram showing the operation of the Time Projection Chamber polarimeter. One dimension of the track images is provided by the array of 128 strips, and the other dimension is provided by the successive samples taken on each strip as the track drifts to the GEM over several time sampling intervals. 


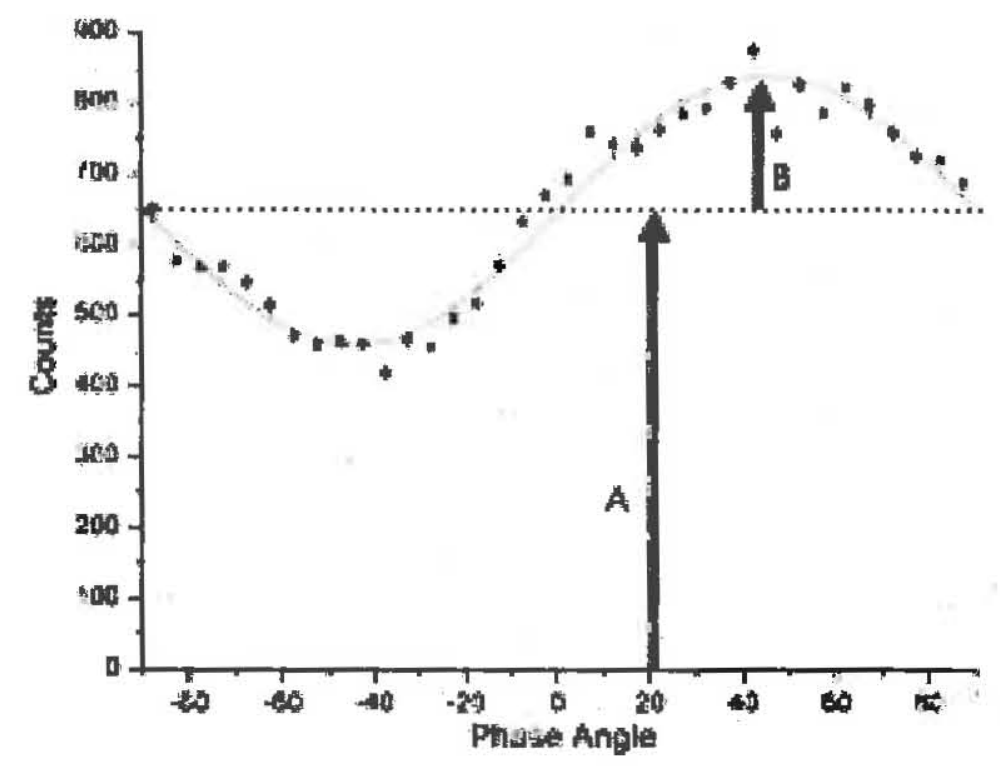

Figure 3. Modulation curve. The modulation factor is given by $\mu=\frac{B}{A}$.

a polarized source is known as a modulation curve, and an example is shown in Figure 3. This curve can be charàcterized with the equation *

$$
N(\theta)=A+B \cos \left[2\left(\theta-\theta_{0}\right)\right] .
$$

The modulation factor, $\mu$, is then defined as

$$
\mu=\frac{B}{A} .
$$

The modulation factor obtained when the polarimeter is observing a source of $100 \%$ polarized X-rays $\left(\mu_{100}\right)$ can be used as a measure of the inherent polarization response of the polarimeter.

Many of the properties and operating parameters of the GEMS polarimeter are collected for reference in Table 1.

\section{SIMULATIONS}

In order to arrive at a better understanding of the working characteristics and output of the GEMS polarimeter, we have constructed a simulation to model the important processes occurring in the detector that shape the output data. This process starts with model tracks generated in DME gas with the Penelope electron transport code. We then model diffusion of the tracks in the drift region, and electron multiplication in the GEM. The ASIC readout impulse response is added to the time series data from the strips, ASIC noise is added to the data, the APV-25 common-mode signal is added, and then simulated pedestals for each strip.

Since the cathode signal is used for many purposes in the instrument, a simulated cathode signal is also generated from the tracks after the gas gain stage. The cathode signal is constructed by summing all the strips in a time sample, applying the cathode impulse response, and then adding an appropriate level of noise. This simulated cathode signal is used to develop and characterize the FPGA algorithms that will be used to provide the trigger signal for the instrument, measure the energy of the event, and implement some of the cuts that are part of the background rejection algorithms.

${ }^{*}$ This $\cos (2 \theta)$ characterization is formally equivalent to the $\cos ^{2}(\theta)$ formalism often seen elsewhere. 


\begin{tabular}{lr}
\hline \hline Energy range & $\overline{2-10 \mathrm{keV}}$ \\
Detector Gas & 190 Torr DME \\
Sampling rate & $\sim 20 \mathrm{MHz}$ \\
Strips & $128 \times 121 \mu \mathrm{m}$ \\
Drift field & $196 \mathrm{~V} / \mathrm{cm}$ \\
GEM field & $500 \mathrm{~V} / 100 \mu \mathrm{m}$ \\
GEM gain & 1500 \\
GEM & $70 \mu \mathrm{m}$ holes \\
& $140 \mu \mathrm{m}$ spacing \\
Pixel size & $121 \mu \mathrm{m}$ pitch \\
Polarimeter active length & $121 \times 121 \mu \mathrm{m}$ \\
Polarimeter active width & $31.2 \mathrm{~cm}$ \\
Polarimeter window diameter & $3 \mathrm{~cm}$ \\
Drift distance (mean) & $14 \mathrm{~mm}$ \\
\hline
\end{tabular}

Table 1. GEMS Polarimeter operating parameters.

The instrument simulations described here have been developed for two main purposes: modeling the performance of the polarimeter by predicting $\mu_{100}$ as a function of energy, and to serve as a testbed for developing and simulating the operation of the onboard science algorithms to be used by the flight FPGA to perform functions such as triggering, energy measurement, windowing, and background rejection.

\subsection{Penelope}

PENELOPE $^{6}$ is a computer software code used for modeling the interactions of electrons with matter. We use it to simulate the path of the initial photoelectron in the DME gas, and the deposition of charge along the track. We have modified Penelope somewhat in order to better model the conditions important for production of tracks in the DME gas used in the GEMS polarimeter. Enhancements to the standard Penelope software include extending the lower energy for tracking electron interactions from $100 \mathrm{eV}$ to about $5 \mathrm{eV}$, and using improved atomic excitation and ionization data for DME. We have turned off all 'soft' interactions in Penelope using the input parameters. We explicitly follow all electrons until their energies fall below the ionization potential of DME. These electrons are then allowed to diffuse as they drift to the gas electron multiplier plane.

The input to the Penelope-based code is the energy, polarization fraction, and polarization angle of the input $\mathrm{X}$-rays, the number of events to simulate, and the parameters of the simulated gas, including the composition, pressure, diffusion coefficients, and drift height. The output is a collection of track images, with each pixel of a track containing the number of primary electrons deposited in the $121 \mu \mathrm{m}$ square pixel.

\subsubsection{Diffusion}

Diffusion of the electrons in the track occurs in the drift region as the track drifts down towards the GEM. The amount of diffusion is dependent on the time taken for the tracks to drift to the GEM; the diffusion can be characterized as going like the square root of the drift time. Longer drift times (from events with initial interactions farther from the GEM) lead to more diffusion, which blur the track and make it more difficult to determine the initial photoelectron direction. This leads to lower modulation factors.

We have used the Magboltz code implemented in the Garfield software package to calculate the transverse and longitudinal diffusion coefficients for DME gas. The transverse and longitudinal diffusion coefficients must be equal to ensure that tracks are not elongated more in one direction than another, which would lead to systematic errors in polarization measurements.

The diffusion coefficients depend on gas parameters such as pressure and on the drift field. The gas pressure and the electric field in the drift region (which determines the drift velocity and affects the diffusion coefficients) 


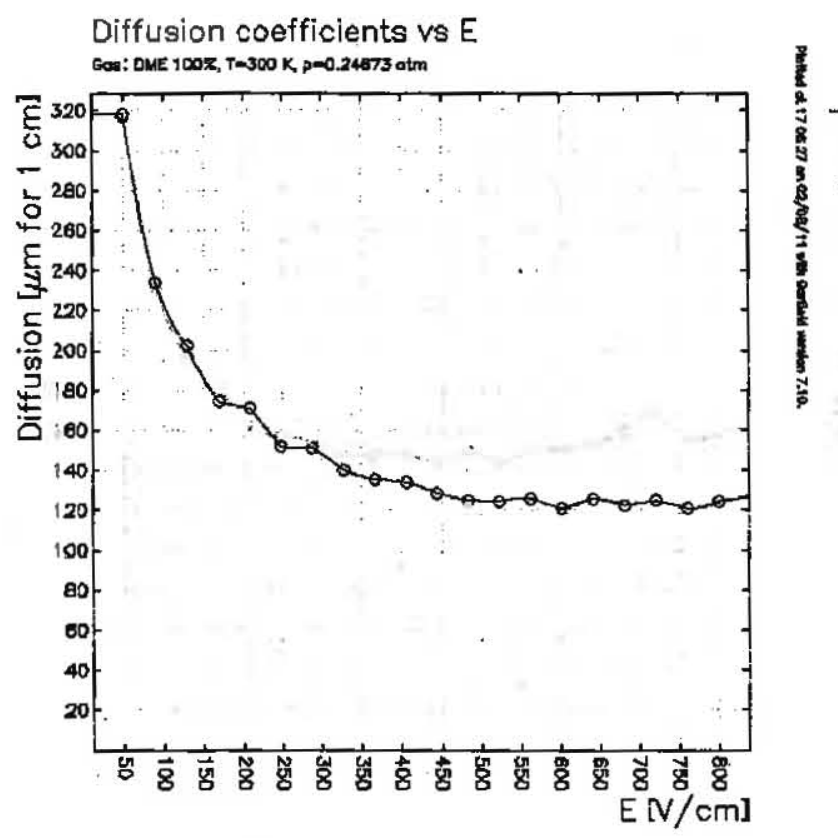

Figure 4. Gas parameters calculated by the Magboltz simulation code for DME at a pressure of 190 Torr. The left panel shows the transverse and longitudinal diffusion coefficients, and the right panel shows the drift velocity as a function of the drift field. In order to reduce systematic errors from non-uniform diffusion, the detector is operated in the regime where the transverse and longitudinal diffusion coefficients are equal but still low, or about $170 \mu \mathrm{m}$ at one $\mathrm{cm}$. This level is reached at a drift field of $196 \mathrm{~V} / \mathrm{cm}$.

\begin{tabular}{lr}
\hline \hline Cathode noise & $2121 \mathrm{e}-$ \\
Cathode impulse response & $60 \mathrm{~ns}$ rise, 1ms fall \\
Boxcar triggering width & 4 samples \\
ASIC response & CR-RC shaping; $\tau=50 \mathrm{~ns}$ \\
Window padding & 2 pixels on each side \\
\hline
\end{tabular}

Table 2. GEMS Polarimeter simulation parameters.

must be carefully chosen in conjunction with the sampling rate in order to ensure square pixels. That is, the product of the drift velocity and the sampling period $(\sim 20 \mathrm{MHz})$ must equal the strip pitch of $121 \mu \mathrm{m}$.

These conditions lead to the nominal detector operating parameters (drift field, sampling rate, gas pressure) listed in Table 1. Figure 4 shows the Magboltz values of the diffusion coefficients as a function of drift field. The longitudinal and transverse diffusion coefficients start to diverge at drift fields higher than $\sim 200 \mathrm{~V} / \mathrm{cm}$, so we adopt the diffusion value at this field ( $170 \mu \mathrm{m}$ for one centimeter of drift) for our simulations. We implement diffusion in the simulations by allowing each electron deposited in the track to diffuse independently to a new position in the image plane.

\subsection{Instrument Simulations in IDL}

After the charge deposition track is generated with Penelope and diffusion added, the rest of the simulation chain is computed using IDL. This includes the gas gain in the GEM, cathode noise and response, ASIC noise and response, cathode triggering and energy measurement, windowing of the track, and deconvolution of the ASIC response. Table 2 lists some of the parameters used in the simulations and described in more detail in the following sections. 


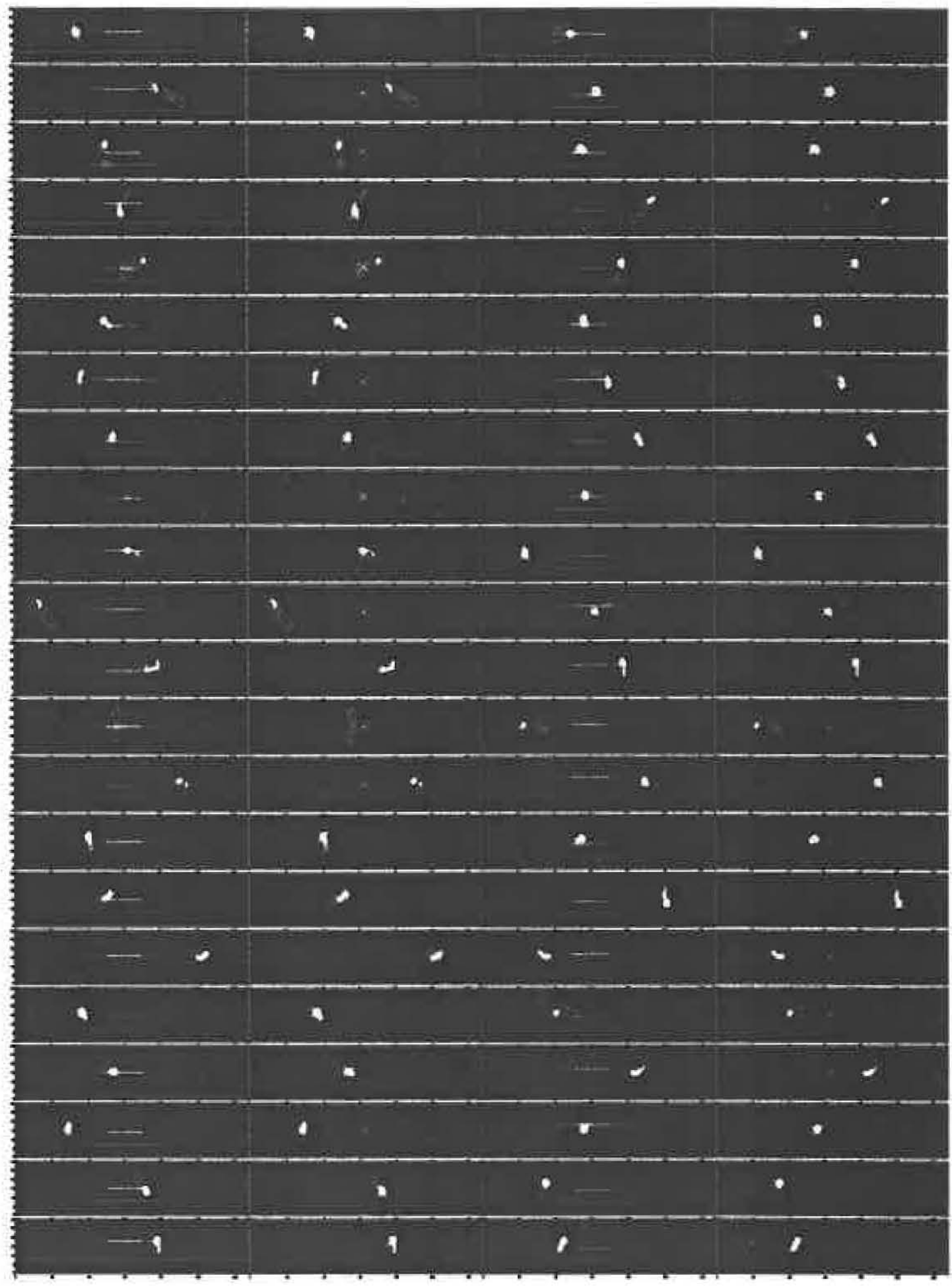

Figure 5. Real Fe-55 $5.9 \mathrm{keV}$ tracks as imaged by the GEMS ETU polarimeter. There are 43 independent X-ray produced tracks shown in this figure; also present is the long, extended and diffuse track of a background event likely caused by a cosmic ray traversing the detector. 


\subsubsection{Cathode signals}

The signal from the cathode side of the GEM is used for multiple purposes in the GEMS polarimeter, including: triggering, energy measurement, drift velocity measurement with the Modulated X-ray Source (MXS), and background rejection.

In the GEMS polarimeter, the cathode signal is amplified with an A250 charge sensitive preamplifier and then digitized for easy manipulation by the onboard flight software.

For simulation purposes, we construct a cathode signal from the Penelope-produced tracks. First, gas gain in the GEM is modeled by applying the standard exponential distribution to each primary electron in the track. We use a nominal gas gain value of 1500 . The cathode signal is then formed by summing together each of the 128 strip samples for each time sampling interval. This cathode signal is then convolved with the cathode preamp impulse response, an exponential function with a $70 \mathrm{~ns}$ rise and $1 \mathrm{~ms}$ fall. Finally, Gaussian noise is added to the signal to match the signal to noise ratio of the A250 preamplifier.

\subsubsection{Triggering}

The triggering algorithm we have devised for GEMS was driven by the need for a robust code relatively insensitive to noise, and one that could be easily implemented by the flight polarimeter FPGA.

The event trigger for GEMS is determined from the sampled cathode preamp signal. The standard algorithm for determining the trigger time will derive the midpoint time of the event. This should work for most signals with energies less than about $8 \mathrm{keV}$. For higher energy events, the cathode signal could be longer than the 30 samples provided by the ASIC. In this case, we will need to use the cathode signal to determine which end of the track is the start and which the finish so we can select the start of the track for retrieval from the ASIC.

The boxcar technique makes a smoothed version of the cathode charge signal and notes when this signal crosses a threshold to determine the trigger times. The triggering algorithm is presented schematically in Figure 6, and in detail below:

Figure 7 illustrates the signals derived from the cathode preamp and used in the triggering process. The top panel shows a simulated track as it would be read out by the ASIC. The next panel shows the simulated cathode preamp signal produced from the image by summing together strips, convolving with the preamp response, and adding noise. The sampled charge signal is produced by taking the difference between successive time samples, and represents the charge present in each time sample of the cathode. The boxcar signal is a smoothed version of the samples charge signal produced by taking a running sum in a 4 sample window and implemented to reduce noise in the sampled charge signal. The crossing of the event threshold by the boxcar signal specifies the start and end of the event, with the trigger time set to the midpoint of the event. The sampled charge signal during the event is saved in an accumulator, which is read out at the end of the event as the measure of the total energy of the event.

Figure 8 shows the triggering performance of the detector as a function of the threshold level. The plot shows the fraction of unsuccessful triggers as a function of the trigger threshold level for three different simulated noise levels and $2 \mathrm{keV}$ events. At high threshold levels, the event signal is not strong enough to cross the threshold and cause a trigger; the threshold level where this occurs is independent of the simulated noise amplitude. At low threshold settings, noise on the cathode signal is large enough to rise above threshold and cause an unsuccessful early trigger. the threshold level where this occurs depends on the simulated noise level, with lower noise levels allowing lower thresholds, as expected. The plot shows that a stable operating threshold level can be set for $2 \mathrm{keV}$ events between these two extremes.

\subsubsection{Energy Resolution}

Figure 9 shows the energy resolution measured from the simulated tracks with the triggering algorithm. This curve behaves with the expected square root dependence of the resolution as a function of energy, and agrees with theoretical predictions made using the known gas properties (Fano factor, energy per electron-ion pair) as well as with measurements made in the lab. 


\section{GEMS Cathode Triggering Algorithm}

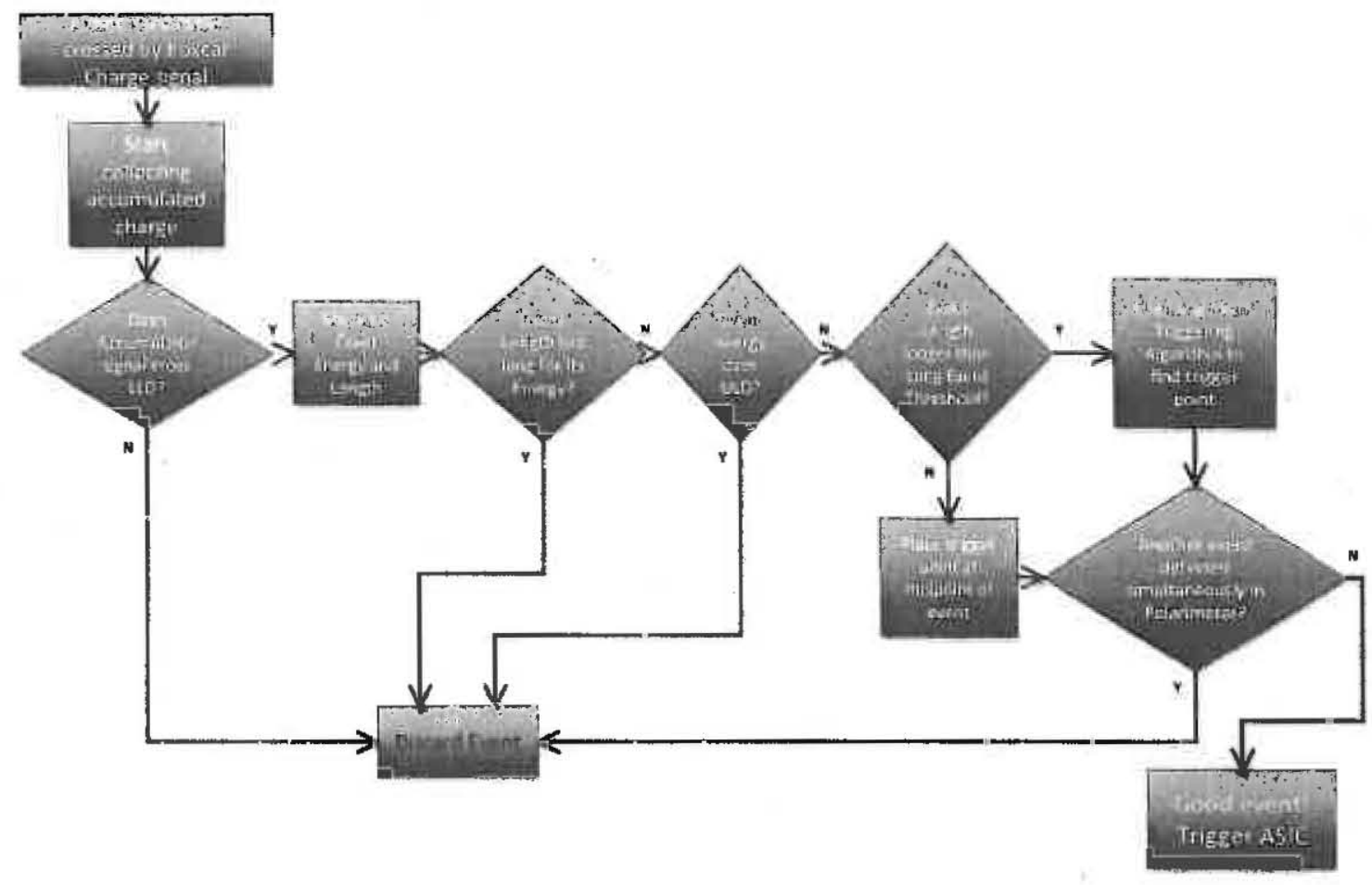

Figure 6. GEMS cathode triggering flowchart.

\subsubsection{Track Lengths}

The track images produced by Penelope are further processed by the simulation to model the operation of the APV25 ASIC. The images are repackaged into the $128 \times 30$ pixel GEMS readout size, the strip signals are convolved with the ASIC impulse response, and noise is added (see Table 2), and FFT deconvolution is done strip by strip using the known ASIC response.

A windowing algorithm locates the center of the track and draws a rectangular box containing all the "hit" pixeis over an imaging threshold. In the instrument this is done in order to significantly reduce the telemetry needed to send the interesting pixels to the ground.

The computed window region for each event can be used to estimate the track lengths of the events in our simulations. We estimate the track length for each event with the length of the diagonal of the rectangular window containing the event as determined by the windowing algorithm. Figure 10 shows several track length distributions as a function of energy. Typical track lengths range from 5 pixels $(605 \mu \mathrm{m})$ at $2 \mathrm{keV}$ to 17 pixels $(2.06 \mathrm{~mm})$ at $10 \mathrm{keV}$. 

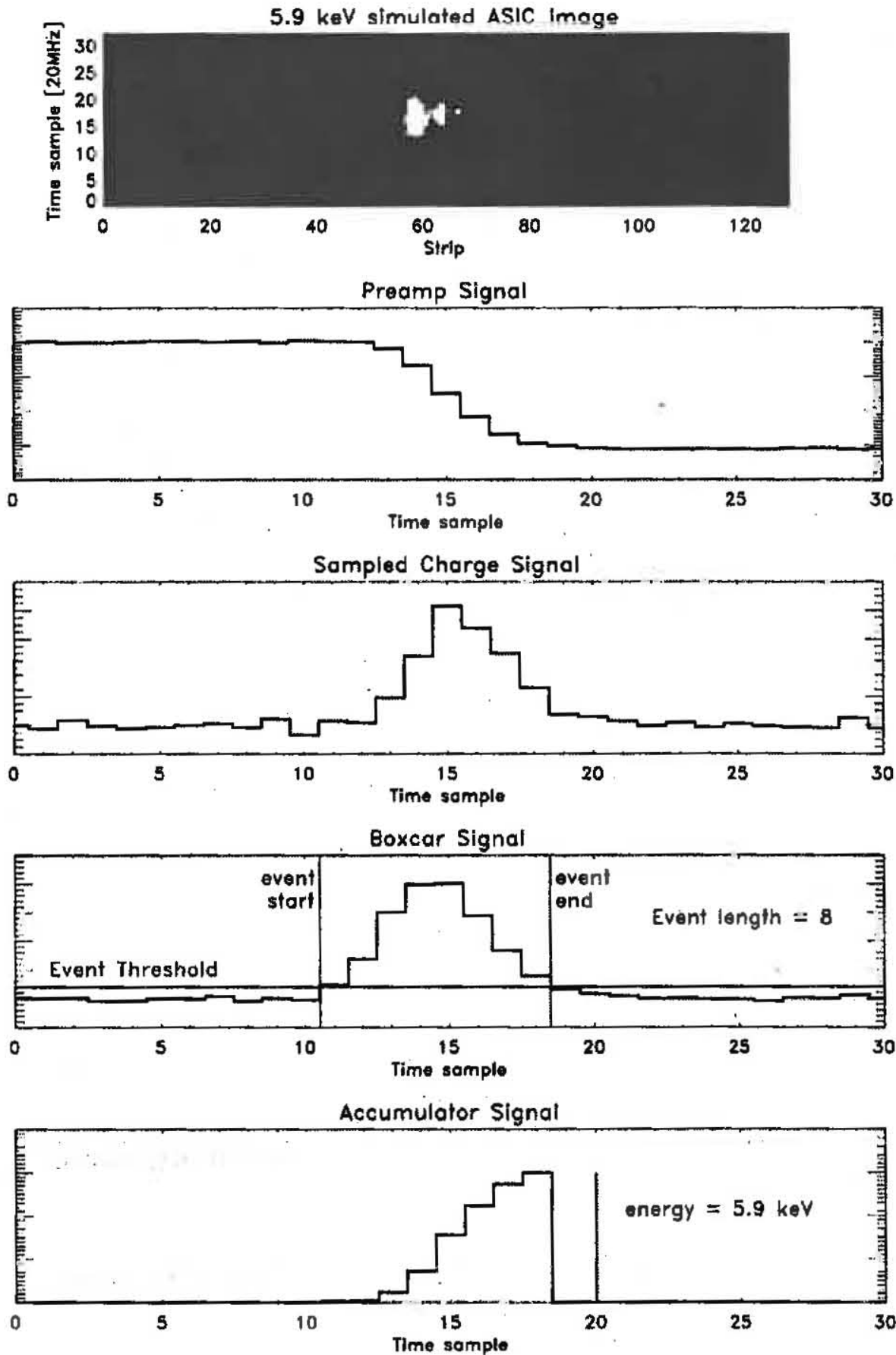

Figure 7. GEMS cathode triggering signals and timing. The simulated preamp signal in the second panel is formed from the track image in the first panel by summing together the strips in a single time sample and convolving with the preamp response. The sampled charge signal is formed by taking the change in the preamp signal from sample to sample, preamp $i-$ preamp $_{i-1}$. The boxcar signal is a smoothed version of the samples charge signal, and is compared with the threshold level to determine the beginning and end of an event trigger. The accumulator signal contains the sum of the sampled charge signal during the trigger, and its value at the end of the trigger is a measure of the energy of the event. 


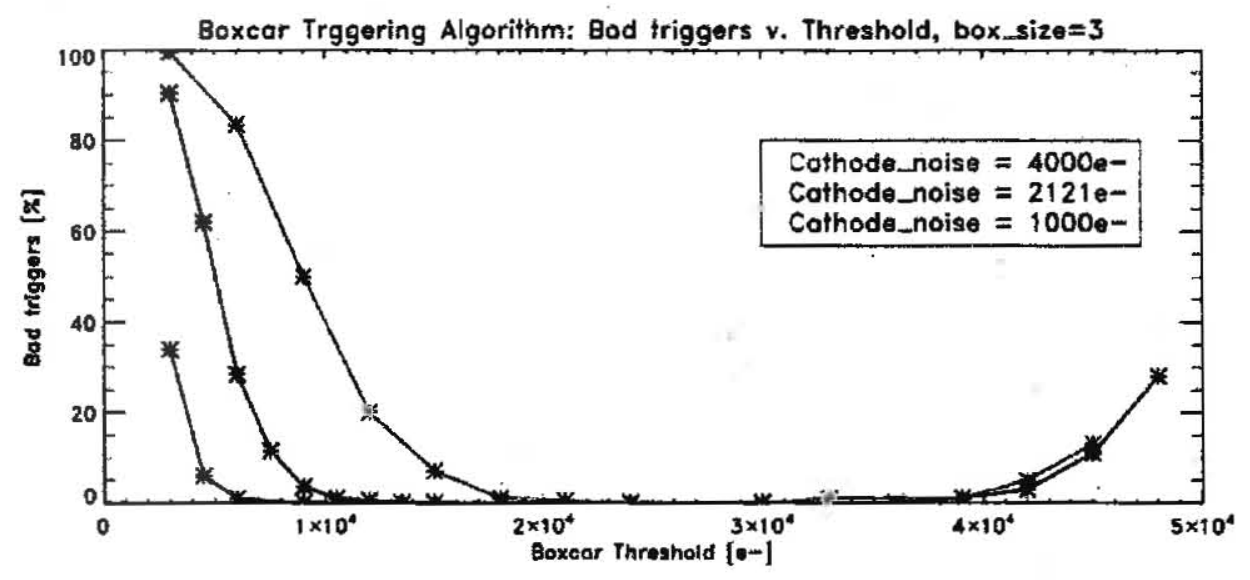

Figure 8. GEMS cathode triggering performance. This plot shows the bad trigger rate (unsuccessful triggers) as a function of trigger threshold for $2 \mathrm{keV}$ events with a boxcar window of three samples. Shown are curves for three modeled cathode nose levels. At low threshold settings, noise above threshold before the event causes a bad trigger. At high thresholds, the signal from the event is not strong enough to cause a trigger.

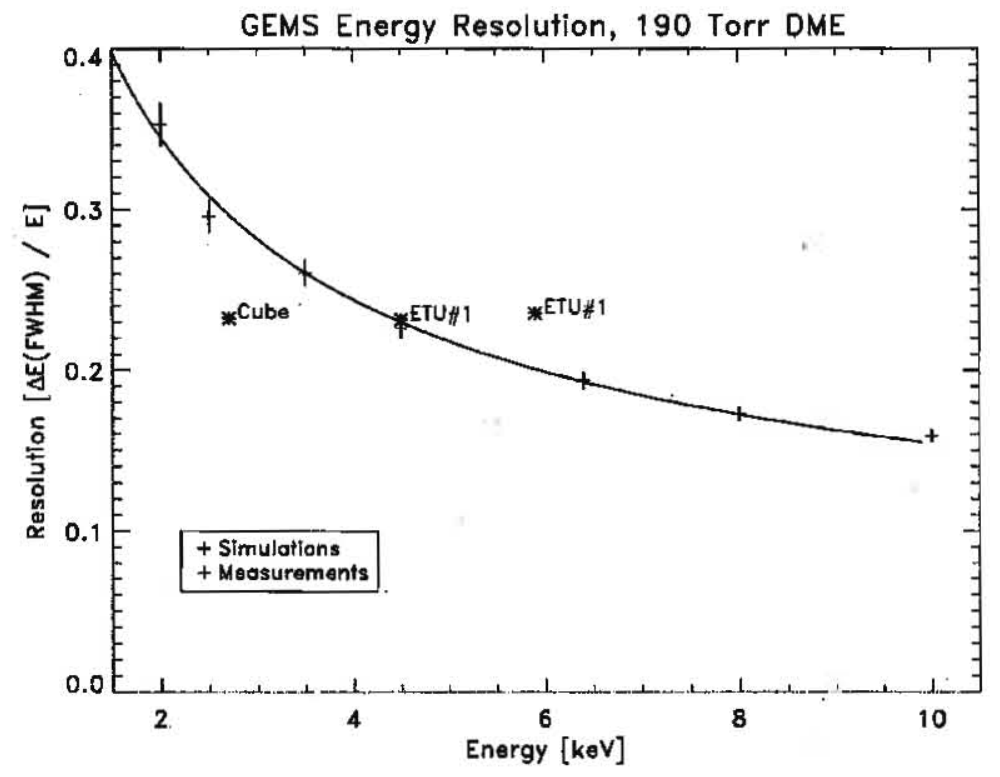

Figure 9. Simulated energy resolution of the GEMS polarimeter. The resolution predicted by the simulations matches theoretical expectations and measurements made with polarimeters in the lab. 


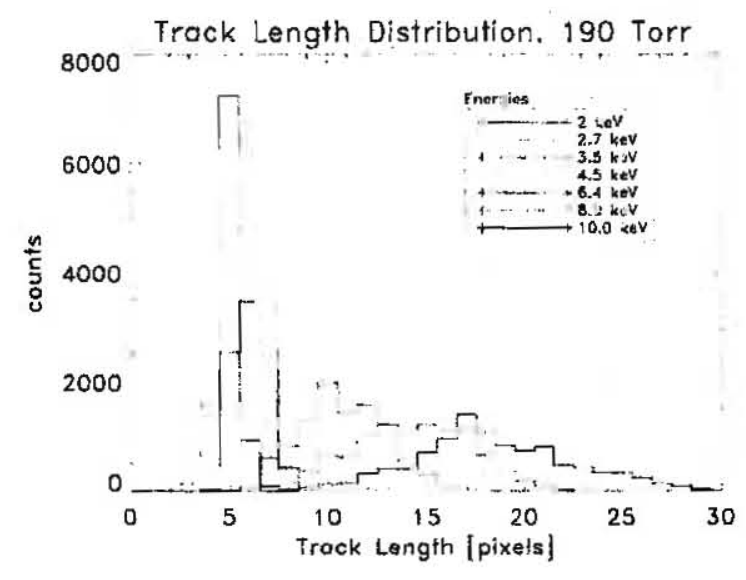

Figure 10. The simulated track length distributions for several different X-ray energies. One pixel is $121 \mu \mathrm{m}$; typical track lengths range from 5 pixels $(605 \mu \mathrm{m})$ at $2 \mathrm{keV}$ to 17 pixels $(2.06 \mathrm{~mm})$ at $10 \mathrm{keV}$.

\section{RECONSTRUCTION}

\subsection{Reconstruction Algorithm}

A reconstruction algorithm is used to determine the direction of the initial photoelectron generated by an X-ray interacting in the polarimeter. We use an algorithm strongly influenced by the one presented in Pacciani et al. $(2003)^{7}$. Our version of this algorithm can be described as a two pass technique: a first estimate of the track direction is made based on the axis of the first moments of the distribution (similar to taking the major axis of an elliptical distribution), and a second pass that attempts to use only the initial part of the track in order to improve the direction estimate.

The reconstruction algorithm is:

1. Calculate the charge barycenter of the track

2. Determine the first moments of the track (including the major and minor axes)

3. Divide the track in two using the minor axis, and find the initial part by finding the half with least charge density

4. Find the most distant pixel from the barycenter in the initial half

5. Search within a limited radius around the most distant pixel for the point zero pixel containing the maximum energy deposit (corresponding to the Auger electron)

6. Calculate the impact point by computing the charge barycenter within a small radius centered on the point zero pixel

7. Calculate the initial track direction from the impact point along a new axis computed from the charge distribution in the first half of the track

The radii we have used to determine the point zero pixel and the impact point are 2-4 pixels; the final track directions to not depend sensitively on these parameters. We have also found that for long tracks ( $>\sim 5 \mathrm{keV}$ ) it is possible to improve the reconstructed track direction by further limiting the portion of the track used in the last step to less than half the size of the full track.

Figure 11 shows shows a $6 \mathrm{keV}$ track image with an illustration of the reconstruction algorithm. The charge barycenter of the event lies at the intersection of the perpendicular lines which represent the first moments of 


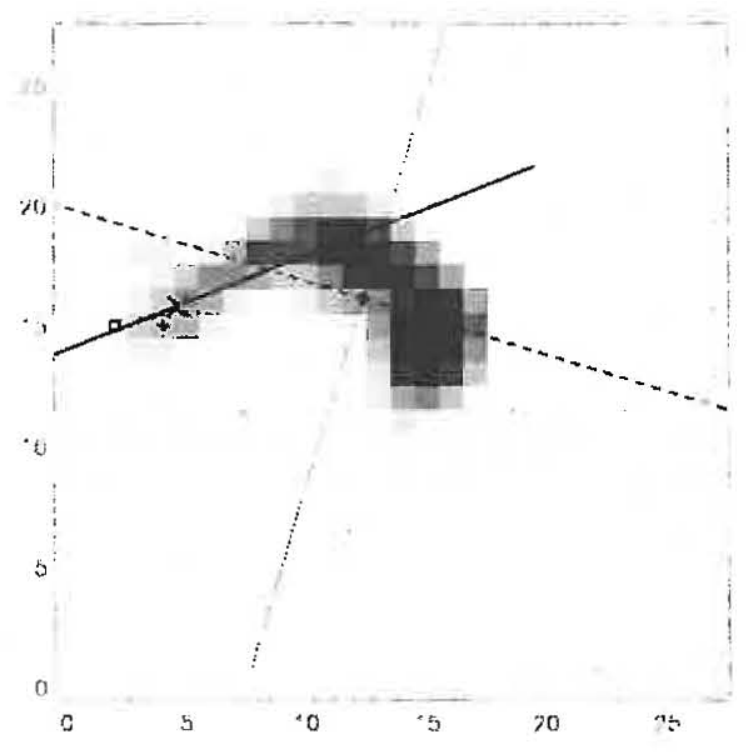

Figure 11. An image of a $6 \mathrm{keV}$ photoelectron track illustrating the reconstruction algorithm. The charge barycenter of the event lies at the intersection of the perpendicular lines which represent the first moments of the charge distribution. The track direction computed by the first pass of the reconstruction algorithm lies along the dotted line. The second pass of the algorithm starts by throwing out the right half of the track, which has a higher charge density indicating the presence of the Bragg peak at the end of the track. The second pass then finds the most distant pixel from the barycenter, represented by the open circle; next finds the point zero pixel (solid dot), the highest charge level within a small radius of the most distant pixel; and then the impact point (" $\mathrm{X}$ ") from the charge barycenter within a small radius of the point zero pixel. The final track direction (solid line through the "X"), is then calculated through the impact point using the charge distribution in the first half of the track.

the charge distribution, the most distant pixel from the barycenter is the open circle, the solid dot is the point zero pixel, and the " $\mathrm{X}$ " is the impact point with the finai reconstructed track direction shown by the dark solid line.

\subsubsection{Eccentricity cuts}

The overall performance of the polarimeter can be improved by discarding nearly circular events that have track directions that are difficult to measure. This comes at the price of reducing the number of events analyzed. The overall performance of the polarimeter can be characterized by the Minimum Detectable Polarization,

$$
M D P \sim \frac{1}{\mu \sqrt{N}}
$$

where $\mu$ is the modulation factor and $N$ the number of counts. This equation holds for the case where background is negligible, as is true for these simulations.

We have run simulations at many energies to determine the optimum eccentricity cut needed to minimize the MDP. In Figure 12, the out-of-roundness is shown on the $\mathrm{x}$-axis (round events on the left and elongated events on the right) with $R$ the ratio of the first and second moments of the track. The y-axis shows the normalized figure of merit (MDP). The figure illustrates that the MDP can be minimized by discarding events with moment ratios less than about 1.2 .

\subsection{Polarimeter modulation factors for $100 \%$ polarized $X$-rays}

A calculation of $\mu_{100}$ as a function of energy for the GEMS polarimeter is one of the main goals of our simulation program. We simulated 200,000 polarized events at each of several energies across the $2-10 \mathrm{keV}$ GEMS passband 


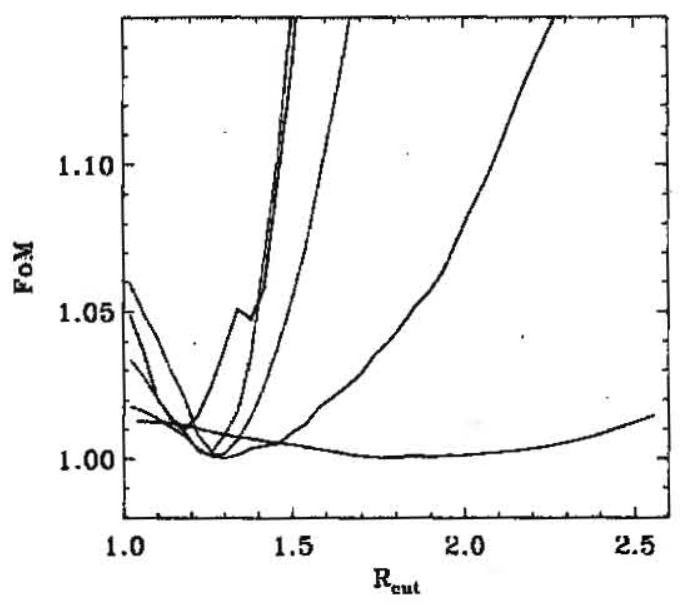

Figure 12. Plot showing the improvement in performance gained by discarding events with small eccentricities. The $\mathrm{x}$-axis is the ratio of the first moments of the charge distribution (rounder events towards the left, longer events to the right), and the $\mathrm{y}$-axis is a figure of merit based on the normalized MDP. The black curve shows $2 \mathrm{keV}$ events, the red curve shows $2.7 \mathrm{keV}$, green is $3.5 \mathrm{keV}$, blue $4.5 \mathrm{keV}$, and black again $6.4 \mathrm{keV}$. The MDP is minimized if events with moment ratios less than 1.2 are discarded.

and reconstructed the photoelectron direction for each simulated track. For simplicity, all events were all simulated with an interaction height of $8 \mathrm{~mm}$ above the GEM, at the midpoint of the detector (we have found by checking a collection of simulations at several drift heights that using the mean height of $8 \mathrm{~mm}$ gives good results). Diffusion was included in the simlations, but not the ASIC response or noise. Uniform rotation of the detector (as required for flight) was also included in the simulation. Modulation curves were computed for each energy, which were then fit to extract modulation factors. All simulated events were kept in the modulation curves, and no eccentricity cuts were made in this simulation. With 200,000 events at each energy, the error on the fit modulation factors was 0.003 .

Figure 13 shows the results of our simulations. At low energies below about $4.5 \mathrm{keV}$ the tracks are more nearly round and are best fit with a one-pass reconstruction. The modulation factor falls off toward lower energies as the tracks become rounder and more difficult to analyze. At higher energies the tracks become longer and the two-pass reconstruction algorithm is used.

The red points in the figure show measurements made at 2.7 and $4.5 \mathrm{keV}$ with polarimeters in the laboratory. These measurements were made using polarized X-ray sources incorporating Bragg reflection from carefully aligned crystals. The laboratory measurements of $\mu_{100}$ for the polarimeter show performance that slightly exceeds that predicted by the simulations. Further investigation will be made of this discrepancy, and is expected to relate to the observation that real tracks from the polarimeter are longer than simulated tracks at the same energy.

Additional confidence in our predicted $\mu_{100}$ values is provided by comparison to independent simulations of the GEMS polarimeter conducted by the RIKEN polarimeter group in Japan. Their simulations used GEANT code to generate tracks instead of Penelope, and produced $\mu_{100}$ values similar to ours to within a few percent over the 2-10 keV band.

\subsection{Attachment coefficient}

Contamination of the gas in the polarimeter can lead to degradation in performance. Contaminants can attach to the free electrons in the drift region, removing a portion of them from the track before they are amplified 


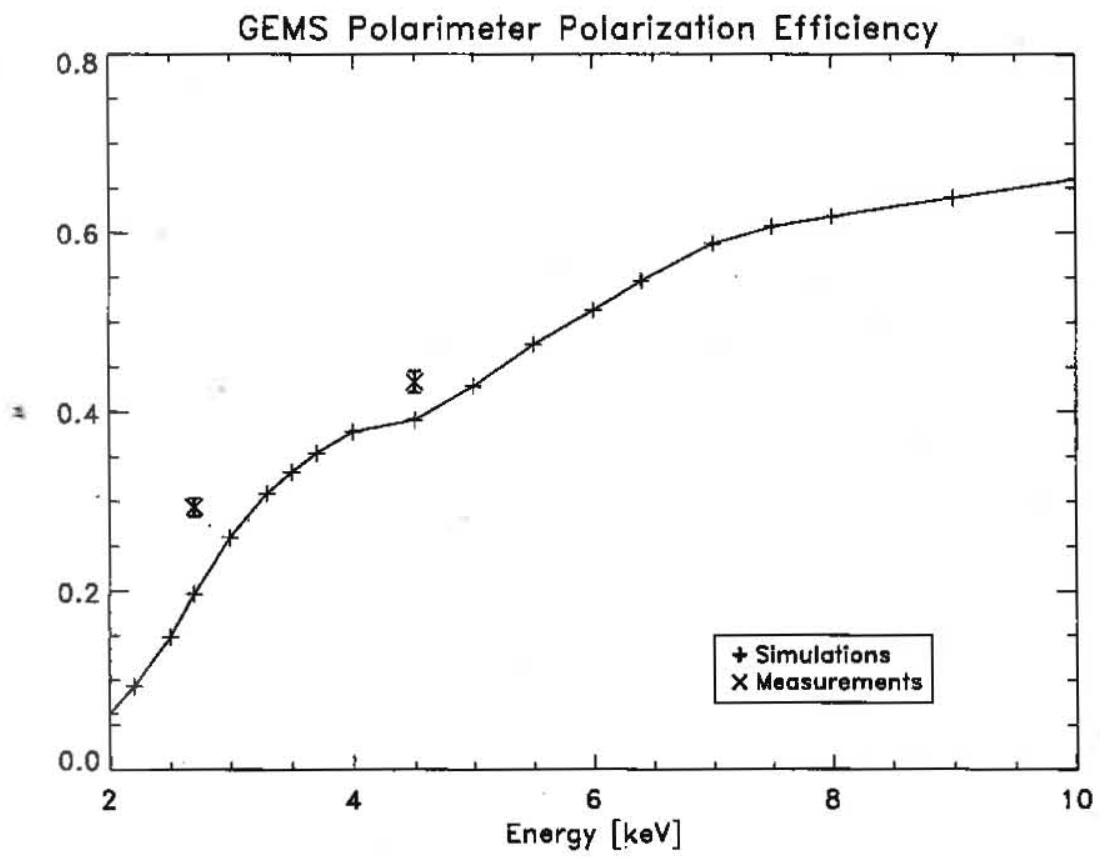

Figure 13. GEMS Polarimeter $\mu_{100}$, or the modulation factor for $100 \%$ polarized X-rays. The crosses connected with solid lines are from simulations, and the points marked with an $\mathrm{X}$ are from laboratory measurements.

in the GEM. This process can be characterized by an attachment coefficient, $\alpha$, that describes how many free electrons deposited in the gas transit the drift region and arrive at the GEM for multiplication:

$$
N(x)=N_{0} e^{-\alpha x},
$$

where $x$ is the drift distance in $\mathrm{cm}$ and $\alpha$ is the attachment coefficient in units of $\mathrm{cm}^{-1}$. The attachment coefficient can be measured and its value in a polarimeter tracked in order to give an estimate of the lifetime of the polarimeter.

We have simulated the response of the polarimeter $\left(\mu_{100}\right)$ to different values of the attachment coefficient. As the attachment coefficient increases and more electrons are removed from the track, the image becomes more sparse and the track direction more difficult to calculate. We have simulated this effect by removing primary electrons from simulated tracks according to the equation above. For simplicity, we ran the simulation for $\mathrm{X}$-rays interacting in the center of the detector with a drift distance of $8 \mathrm{~mm}$.

Our results are shown in Figure 14. The modulation factor does not change rapidly with increasing values of the attachment coefficient. An attachment coefficient of 2.5 indicates the free electrons travel through two scale lengths in the drift region and that nearly $90 \%$ of the initial electron are lost. Even in this drastic case, modulation factors at $6.4 \mathrm{keV}$ have only fallen by 0.10 from their initial values with zero attachment coefficient.

\section{CONCLUSIONS}

We have developed a Penelope-based simulation code in order to predict the performance of the GEMS X-ray Polarimeter. We have generated track images at several energies in the 2-10 keV GEMS passband and have analyzed them with our track reconstruction code in order to determine the direction of the initial photoelectron from the X-ray interaction, make modulation curves, and determine the modulation factor of the polarimeter for $100 \%$ polarized X-rays. These predicted modulation factors range from $10-60 \%$ over $2-10 \mathrm{keV}$, and are consistent with actual polarimeter measurements taken in the laboratory of $100 \%$ polarized calibration sources. 


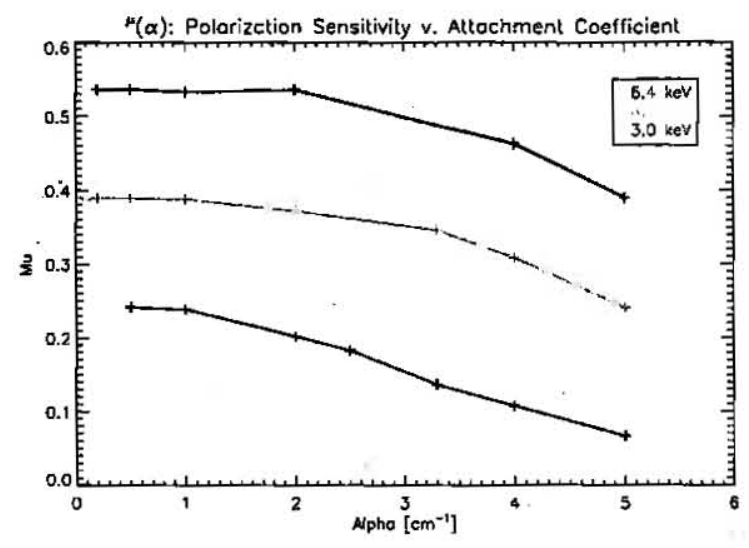

Figure 14. Modulation factor versus attachment coefficient for several energies.

A related simulation to investigate the effects of contamination in the detector gas by modeling the loss of electrons due to a non-zero attachment coefficient finds that the modulation factor drops only modestly even for attachment coefficients large enough to cause the loss of over half the electrons in the drift region. This can be understood qualitatively by considering that even the loss of significant numbers of electrons from the track image still leaves enough of its overall shape present to enable adequate determination of the track direction.

We have also modeled the operational characteristics of the GEMS polarimeter in order to develop and. characterize the behavior of the flight algorithms used for triggering, energy measurement, windowing, and background rejection, and present simulated energy resolution and track length measurements.

\section{ACKNOWLEDGMENTS}

We would like to thank C.J. Martoff for his work modifying the Penelope code for improved use in simulating GEMS photoelectron tracks, and G.S. and S.W. for help with the manuscript of this paper.

\section{REFERENCES}

1 Black, J.K., et al. "X-ray polarimetry with a micropattern TPC", NIM A, 581, 755-760 (2007)

2 Black, J.K., et al. "TPCs in high-energy astronomical polarimetry", 3rd Symposium on Large TPCs for Low Energy Rare Event Detection, Journal of Physics: Conference Series 65012005 (2007)

3 Black, J.K., et al. "The GEMS Photoelectric X-ray Polarimeters", Proc. SPIE 7732, pp. 77320X-77320X$11(2010)$

4 Hill, J., et al. "The GEMS Photoelectric X-ray Polarimeters", Proc. SPIE 8443(2012)

5 Swank, J., Kallman, T., Jahoda, K., Black, K., Deines-Jones, P., and Kaaret, P. "Gravity and Extreme Magnetisn SMEX (GEMS)" in X-Ray Polarimetry: A New Window in Astrophysics, ed. R. Bellazzini , E. Costa, G. Matt and G. Tagliaferri (New York: Cambridge University Press), p. 251 (2010)

6 J. Sempau, E. Acosta, J. Baro, J.M. Fernandez-Varea and F.Salvat "An algorithm for Monte Carlo simulation of the coupled electron-photon transport", Nuclear Instruments and Methods B 132 (1997) 377-390.

7 Pacciani et al. "The sensitivity of a photoelectric X-ray polarimeter for Astronomy: The impact of gas mixture and pressure" Proc. SPIE 4843, 394 (2003) 
